CRYSTALLOGRAPHIC COMMUNICATIONS

ISSN 2056-9890

Received 18 July 2019

Accepted 20 July 2019

Edited by M. Zeller, Purdue University, USA

Keywords: crystal structure; ligand; dithiolene; metalloligand.

CCDC reference: 1941888

Supporting information: this article has supporting information at journals.iucr.org/e

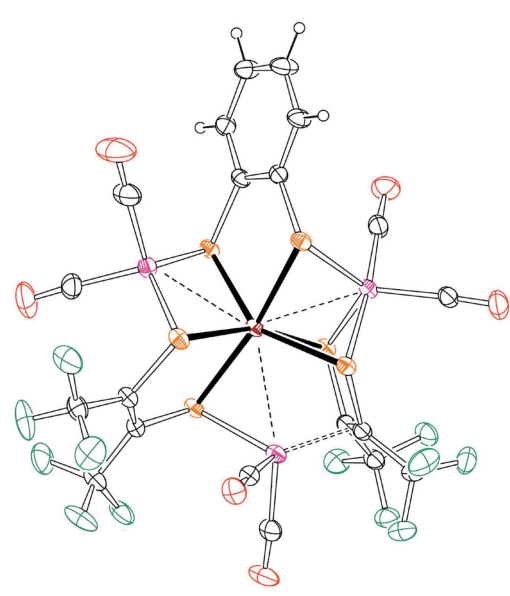

OPEN $\odot$ ACCESS

\section{A molybdenum tris(dithiolene) complex coordinates to three bound cobalt centers in three different} ways

\author{
Neilson Nguyen, ${ }^{a}$ Alan J. Lough ${ }^{\mathrm{b}}$ and Ulrich Fekl ${ }^{\mathrm{a} *}$
}

\begin{abstract}
${ }^{\mathrm{a}}$ Department of Chemical and Physical Sciences, University of Toronto Mississauga, 3359 Mississauga Rd, Mississauga, Ontario, L5L 1C6, Canada, and ${ }^{\mathbf{b}}$ Department of Chemistry, University of Toronto, 80 St. George St., Toronto, Ontario, M5S 3H6, Canada. *Correspondence e-mail: ulrich.fekl@utoronto.ca
\end{abstract}

The synthesis and structural characterization of the molecular compound $\left(\mu_{3^{-}}\right.$ benzene-1,2-dithiolato)hexacarbonylbis $\left(\mu_{3}-1,1,1,4,4,4\right.$-hexafluorobut-2-ene-2,3dithiolato)tricobaltmolybdenum, $\left[\mathrm{Co}_{3} \mathrm{Mo}\left(\mathrm{C}_{4} \mathrm{~F}_{6} \mathrm{~S}_{2}\right)_{2}\left(\mathrm{C}_{6} \mathrm{H}_{4} \mathrm{~S}_{2}\right)(\mathrm{CO})_{6}\right]$ or $\mathrm{Mo}(\mathrm{tfd})_{2^{-}}$ (bdt) $\left(\mathrm{Co}(\mathrm{CO})_{2}\right)_{3}$ (tfd is 1,1,1,4,4,4-hexafluorobut-2-ene-2,3-dithiolate and bdt is benzene-1,2-dithiolate), are reported. The structure of the molecule contains the molybdenum tris(dithiolene) complex $\mathrm{Mo}(\mathrm{tfd})_{2}(\mathrm{bdt})$ coordinated as a multidentate ligand to three cobalt dicarbonyl units. Each of the three cobalt centers is relatively close to molybdenum, with Co *.Mo distances of 2.7224 (7), 2.8058 (7), and 2.6320 (6) $\AA$. Additionally, each of the cobalt centers is bound via main-group donor atoms, but each one in a different way: the first cobalt atom is coordinated by two sulfur atoms from different dithiolenes (bdt and tfd). The second cobalt atom is coordinated by one sulfur from one tfd and two olefinic carbons from another tfd. The third cobalt is coordinated by one sulfur from bdt and two sulfurs from tfd. This is, to the best of our knowledge, the first structurally characterized example of a molybdenum (tris)dithiolene complex that coordinates to cobalt. The $\mathrm{F}$ atoms of two of the $-\mathrm{CF}_{3}$ groups were refined as disordered over two sets of sites with ratios of refined occupancies of 0.703 (7):0.297 (7) and $0.72(2): 0.28(2)$.

\section{Chemical context}

Sulfur removal from crude petroleum is performed on a large industrial scale through a process called hydrodesulfurization. This involves use of hydrogen gas (the sulfur is removed as $\mathrm{H}_{2} \mathrm{~S}$ ) and addition of a catalyst, typically cobalt-doped $\mathrm{MoS}_{2}$ (Hinnemann et al., 2008). $\mathrm{MoS}_{2}$ is not a molecular compound but rather possesses an extended structure, consisting of closepacked sulfur layers between which molybdenum is sandwiched (Dickinson \& Pauling, 1923). The coordination geometry around molybdenum is trigonal prismatic. Several attempts to model $\mathrm{MoS}_{2}$ using molecular compounds have been made, often using dithiolene $\left(\mathrm{S}_{2} \mathrm{C}_{2} R_{2}\right)$ ligands, where some examples also contain the hydrodesulfurization-relevant addition of cobalt. Complexes containing molybdenum, cobalt, and one or two (but not three) dithiolenes are known. A molybdenum bis(dithiolene) that coordinates to two cobalt centers has been characterized crystallographically (Nihei $e t$ al., 1999; Murata et al., 2006). The study reports $\left[\mathrm{Mo}(\mathrm{bdt})_{2}(-\right.$ $\left.\mathrm{CO})_{2}(\mathrm{CpCo})_{2}\right]$, where bdt $=o-\mathrm{C}_{6} \mathrm{H}_{4} \mathrm{~S}_{2}$ and $\mathrm{Cp}=$ cyclo $-\mathrm{C}_{5} \mathrm{H}_{5}$. The methylated analog $\left[\mathrm{Mo}(\mathrm{bdt})_{2}(\mathrm{CO})_{2}(\mathrm{Cp} * \mathrm{Co})_{2}\right]$, where $\mathrm{Cp}^{*}$ $=$ cyclo $-\mathrm{C}_{5} \mathrm{Me}_{5}$ was also structurally characterized (Muratsugu et al., 2011). An analogous $\left[\mathrm{Mo}(\mathrm{ddds})_{2}(\mathrm{CO})_{2}(\mathrm{CpCo})_{2}\right]$ was reported by a different group, where ddds is the unusual di- 
thiolene 1,2-dicarba-closo-dodecaborane-1,2-disulfide (Chen et al., 2007). The above contribution also reported a molybdenum mono(dithiolene) complex coordinated to a cobalt fragment, namely $\left[\mathrm{Mo}(\mathrm{ddds})(\mathrm{CO})_{2}(\mathrm{py})_{2}(\mathrm{Cp} * \mathrm{Co})\right]$, where py $=$ pyridine. Coordinating to cobalt a molybdenum tris(dithioene), that is a compound where three dithiolenes are bound to molybdenum, would be interesting, because molybdenum tris(dithiolene)s mimic $\mathrm{MoS}_{2}$ particularly well. Similar to $\mathrm{MoS}_{2}$, they contain molybdenum coordinated to six sulfur atoms, and, also, depending on the oxidation state of the compound, the environment of molybdenum can sometimes be trigonal prismatic (Beswick et al., 2004). However, we could not find any structurally characterized example for how a molybdenum tris(dithiolene) complex can act as ligand for cobalt. Such an example is provided here. In 2007, the mixed dithiolene complex $\mathrm{Mo}(\mathrm{tfd})_{2}(\mathrm{bdt})\left[\mathrm{tfd}=\mathrm{S}_{2} \mathrm{C}_{2}\left(\mathrm{CF}_{3}\right)_{2}\right]$, an unsymmetrical tris(dithiolene), was reported for the first time (Harrison et al., 2007). Later, this complex, which contains two different dithiolenes, was used to create structural models for the active sites of $\mathrm{MoS}_{2}$ hydrodesulfurization catalysts, albeit cobalt-free ones (Nguyen et al., 2010). In this current work, we have successfully linked three cobalt centers to one $\mathrm{Mo}(\mathrm{tfd})_{2}$ (bdt) molecule. Surprisingly, each of the three cobaltcontaining units [each one is a $\mathrm{Co}(\mathrm{CO})_{2}$ fragment] is bound to the molybdenum tris(dithiolene) center in a different way.

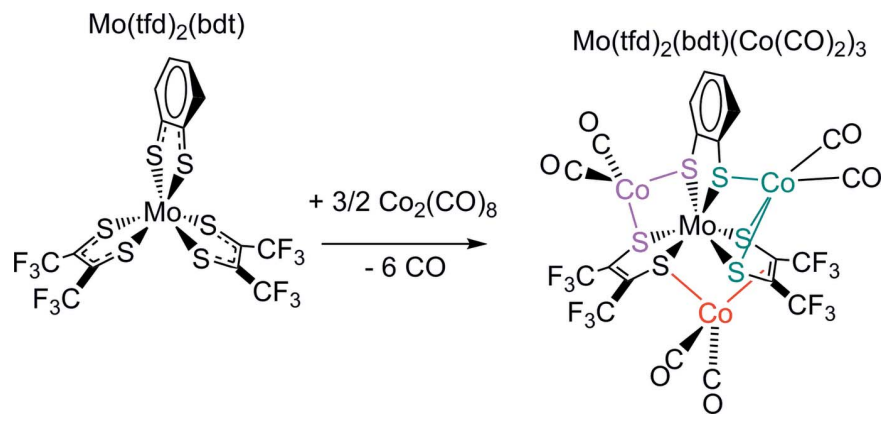

\section{Structural commentary}

An anisotropic displacement plot showing the structure of the $\mathrm{Mo}(\mathrm{tfd})_{2}(\mathrm{bdt})\left(\mathrm{Co}(\mathrm{CO})_{2}\right)_{3}$ molecule is shown in Fig. 1. A good starting point for the structural description is considering the core $\mathrm{Mo}(\mathrm{tfd})_{2}(\mathrm{bdt})$ substructure first. Molybdenum is coordinated by six sulfur atoms, four from the two tfd ligands, two from the one bdt ligand. The $\mathrm{Mo}-\mathrm{S}$ distances are fairly normal, ranging from 2.413 (1) $\AA$ (Mo1-S5) to 2.457 (1) $\AA$ (Mo1-S4). The appearance of the structure is 'approximately octahedral'. A more quantitative measure is obtained using the $X-M-X_{\text {trans }}$ criterion (Beswick et al., 2004; Nguyen et al., 2010), which indicates that the geometry around molybdenum is $71 \%$ octahedral ( $29 \%$ trigonal prismatic). The intra-ring $\mathrm{C}-\mathrm{C}$ distance in the tfd ligand that is not $\pi$-coordinated to cobalt (C5-C6) is 1.335 (6) $\AA$, indicating that description as an ene-dithiolate is appropriate (Hosking et al., 2009). The intra-ring $\mathrm{C}$-C distance in the tfd ligand that is $\pi$-coordinated to cobalt $(\mathrm{C} 1-\mathrm{C} 2)$ is much longer, at 1.439 (6) $\AA$, but this elongation is expected as an effect of $\pi$-coordination to cobalt

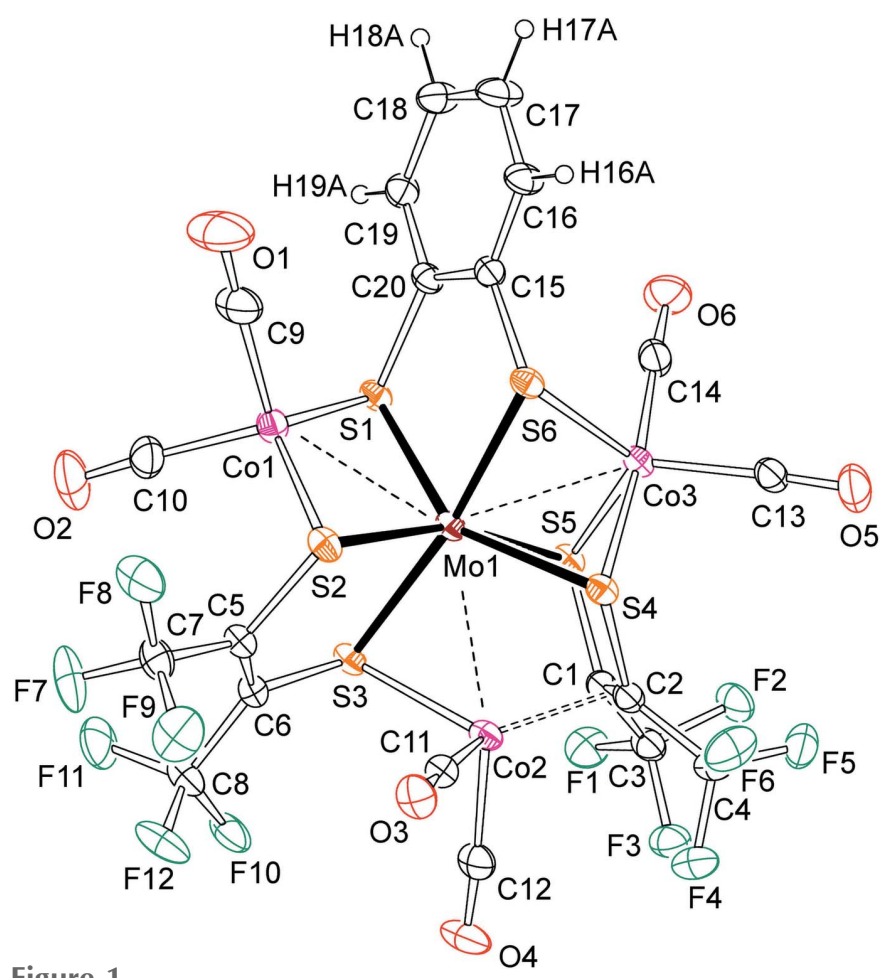

Figure 1

A view of the molecular structure of $\mathrm{Mo}(\mathrm{tfd})_{2}(\mathrm{bdt})\left(\mathrm{Co}(\mathrm{CO})_{2}\right)_{3}$. Anisotropic displacement ellipsoids in this plot, generated with ORTEP-3 (Farrugia, 2012), are shown at the 30\% level. Hydrogen atoms are shown as spheres of arbitrary radius. For the disordered fluorines on C7 and C8 only one orientation (major component) is shown.

(Co2). At this point it makes sense to discuss the way in which the $\mathrm{Mo}(\mathrm{tfd})_{2}$ (bdt) substructure coordinates to the three cobalt dicarbonyl fragments. Co1 is coordinated by two sulfurs from different dithiolenes (bdt and tfd) at bond lengths of 2.225 (1) $\AA$ (Co1-S1) and 2.241 (1) $\AA(\mathrm{Co} 1-\mathrm{S} 2)$, respectively. The $\mathrm{C}_{2} \mathrm{~S}_{2}$ environment of $\mathrm{Co} 1$ is nearly tetrahedral, with very slight distortions. The $\mathrm{S} 1-\mathrm{Co} 1-\mathrm{S} 2$ angle is slightly wide, at $115.44(5)^{\circ}$, the $\mathrm{C} 9-\mathrm{Co} 1-\mathrm{C} 10$ angle is slightly narrow, at $98.4(3)^{\circ}$. Co2, in contrast, is coordinated by one sulfur from one tfd and two olefinic carbons from another tfd, where bond lengths are $2.256(1) \AA(\mathrm{Co} 2-\mathrm{S} 3), 2.010(4) \AA(\mathrm{Co} 2-\mathrm{C} 1)$, and $1.970(4)(\mathrm{Co} 2-\mathrm{C} 2)$. The coordination geometry of $\mathrm{Co} 2$ (not including Mo1 here) is, again, approximately tetrahedral, where the largest deviation from tetrahedral geometry are $\mathrm{C} 11-\mathrm{Co} 2-\mathrm{C} 12$, at $96.3(2)^{\circ}$ and the comparably wide 'bite' of the chelating substructure, with $C t 1-\mathrm{Co} 2-\mathrm{S} 3$ measuring $119.7^{\circ}$, where $C t 1$ is the mid-point between $\mathrm{C} 11$ and $\mathrm{C} 12$. Another sulfur atom, S4, is relatively close to $\mathrm{Co} 2$, but the interatomic distance, at 2.767 (1) $\AA$, is considerably longer than the $\mathrm{Co} 2-\mathrm{S} 3$ bond, such that S4 is almost certainly not bonded. Finally, $\mathrm{Co} 3$ is coordinated by one sulfur from bdt and two sulfurs from tfd, at distances of 2.234 (1) $\AA$ (Co3-S4), 2.239 (1) $\AA$ (Co3-S5), and 2.275 (1) $\AA$ (Co3-S6). Co3 is surrounded by these three sulfurs and two carbons in an approximately trigonal-bipyramidal fashion. C14 and S4 occupy axial positions, with the C14-Co3-S4 angle being $174.3(2)^{\circ}$. The three angles in the trigonal plane are $115.38(4)^{\circ}(\mathrm{S} 5-\mathrm{Co} 3-\mathrm{S} 6), 118.6(2)^{\circ}(\mathrm{S} 5-\mathrm{Co} 3-\mathrm{C} 13)$, and 
Table 1

Hydrogen-bond geometry $\left(\AA,^{\circ}\right)$.

\begin{tabular}{lllll}
\hline$D-\mathrm{H} \cdots A$ & $D-\mathrm{H}$ & $\mathrm{H} \cdots A$ & $D \cdots A$ & $D-\mathrm{H} \cdots A$ \\
\hline $\mathrm{C} 17-\mathrm{H} 17 A \cdots \mathrm{O}^{\mathrm{i}}$ & 0.95 & 2.57 & $3.353(7)$ & 140 \\
$\mathrm{C} 17-\mathrm{H} 17 A \cdots \mathrm{F} 11^{\mathrm{ii}}$ & 0.95 & 2.62 & $3.461(9)$ & 148 \\
$\mathrm{C} 17-\mathrm{H} 17 A \cdots \mathrm{F} 10 A^{\mathrm{ii}}$ & 0.95 & 2.55 & $3.29(2)$ & 135 \\
\hline
\end{tabular}

Symmetry codes: (i) $-x+1,-y+2,-z$; (ii) $x+\frac{1}{2}, y+\frac{1}{2}, z$.

$124.0(2)^{\circ}(\mathrm{S} 6-\mathrm{Co} 3-\mathrm{C} 13)$. While there is no doubt that the three cobalt atoms are bound by the heteroatoms (sulfur, carbon) of the $\mathrm{Mo}(\mathrm{tfd})_{2}(\mathrm{bdt})$ structure, each of the three cobalt atoms is also close to the central molybdenum, and these metal-metal contacts could possibly be bonding as well. The relevant distances are 2.7224 (7) $\AA$ (Co1-Mo1),

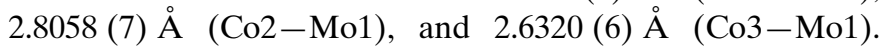
Such Mo-Co distances are typically considered of a range compatible with Mo-Co bonds (Chen et al., 2007; Curtis et al., 1997; Murata et al., 2006; Muratsugu et al., 2011).

\section{Supramolecular features}

Molecules of $\mathrm{Mo}(\mathrm{tfd})_{2}(\mathrm{bdt})\left(\mathrm{Co}(\mathrm{CO})_{2}\right)_{3}$ pack, without any solvent in the crystal, via contacting van der Waals surfaces. The packing pattern is shown in Fig. 2. Hydrogen atom H17 $A$ forms close intermolecular contacts to an oxygen atom from a neighboring carbonyl and to a fluorine atom of the major disorder component (F11), as well as to a fluorine atom of the minor disorder component $(\mathrm{F} 10 A)$. Details can be found in Table 1.

\section{Database survey}

The Cambridge Crystallographic Database (version 5.40, including updates up to May 2019; Groom et al., 2016) was searched. The search was performed as a substructure search containing the most general dithiolene $\mathrm{S}-\mathrm{C}-\mathrm{C}-\mathrm{S}$ substructure

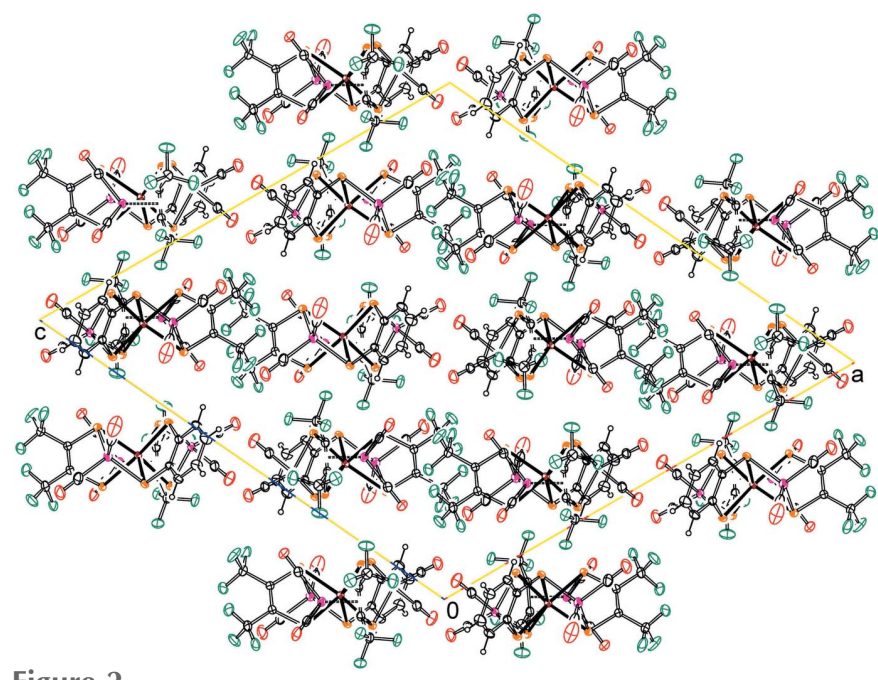

Figure 2

Packing of molecules of $\mathrm{Mo}(\mathrm{tfd})_{2}(\mathrm{bdt})\left(\mathrm{Co}(\mathrm{CO})_{2}\right)_{3}$, viewed along the $b$ axis. (with any kind of bond allowed in the chain), plus a molybdenum and a cobalt atom. Since no specific requirement was imposed with regard to whether or in which way cobalt or molybdenum are bonded to the $\mathrm{S}-\mathrm{C}-\mathrm{C}-\mathrm{S}$ structure, hits that do not contain a molybdenum dithiolene complex coordinated to cobalt where manually removed as follows. Seven hits were retrieved: EYUHIQ, JOQWIV, JOQWIV01, SEVMIQ, SEVMOW, TASDAT, OQAMEZ. Out of these, TASDAT and OQAMEZ are not relevant here, since they do not contain a molybdenum dithiolene unit that is directly bonded to cobalt. They contain, respectively, a cobalt-based counter-cation for an anionic molybdenum complex and a nickel bis(dithiolene) anion as a counter-anion for a molybdenum/cobalt sulfido cluster. The structures EYUHIQ, JOQWIV, JOQWIV01, SEVMIQ and SEVMOW are relevant, since they contain at least one molybdenum dithiolene unit that is directly bonded to cobalt. These structures are all discussed above in the Chemical context (Nihei et al., 1999; Murata et al., 2006; Muratsugu et al., 2011; Chen et al., 2007).

\section{Synthesis and crystallization}

$\mathrm{Mo}(\mathrm{tfd})_{2}(\mathrm{bdt})\left(\mathrm{Co}(\mathrm{CO})_{2}\right)_{3}$ was prepared from $\mathrm{Mo}(\mathrm{tfd})_{2}(\mathrm{bdt})$ (Harrison et al., 2007) and dicobaltoctacarbonyl (obtained from Sigma-Aldrich) as summarized in the Scheme, using airfree conditions and rigorously dried solvents. $48 \mathrm{mg}$ of $\mathrm{Mo}(\mathrm{tfd})_{2}(\mathrm{bdt})(0.0697 \mathrm{mmol})$ were dissolved in $18 \mathrm{~mL}$ of hexane (dried over $\mathrm{Na} /$ benzophenone). $60 \mathrm{mg}(0.175 \mathrm{mmol})$ of $(\mathrm{Co})_{2}(\mathrm{CO})_{8}$ dissolved in $2 \mathrm{~mL}$ of hexane were added and the mixture was shaken. The mixture was stored overnight at $243 \mathrm{~K}$ in the freezer of a nitrogen-filled glovebox. The supernatant was decanted off, and the black crystals of $\mathrm{Mo}(\mathrm{tfd})_{2}(\mathrm{bdt})\left(\mathrm{Co}(\mathrm{CO})_{2}\right)_{3}$ were washed twice with $5 \mathrm{~mL}$ of cold hexane. Total yield $27 \mathrm{mg}(0.026 \mathrm{mmol}, 37 \%)$. Analysis calculated for $\mathrm{Mo}_{1} \mathrm{~S}_{6} \mathrm{C}_{20} \mathrm{H}_{4} \mathrm{~F}_{12} \mathrm{Co}_{3} \mathrm{O}_{6}$ : C, 23.25; $\mathrm{H}, 0.39 ; \mathrm{O}, 9.29$; $\mathrm{S}, 18.62$. Found: C, 23.70; H, 0.44; O, 9.70; S, 18.80. ${ }^{1} \mathrm{H}$ NMR $\left(400 \mathrm{MHz}, \mathrm{C}_{6} \mathrm{D}_{6}\right): \delta 6.38(\mathrm{~m}), 7.02(\mathrm{~m})$. The compound is paramagnetic. An estimate of the magnetic moment in solution (Evans method) yielded $c a 0.9 \mathrm{BM}$, consistent with one unpaired electron. An EPR spectrum was also obtained, shown in Fig. 3.

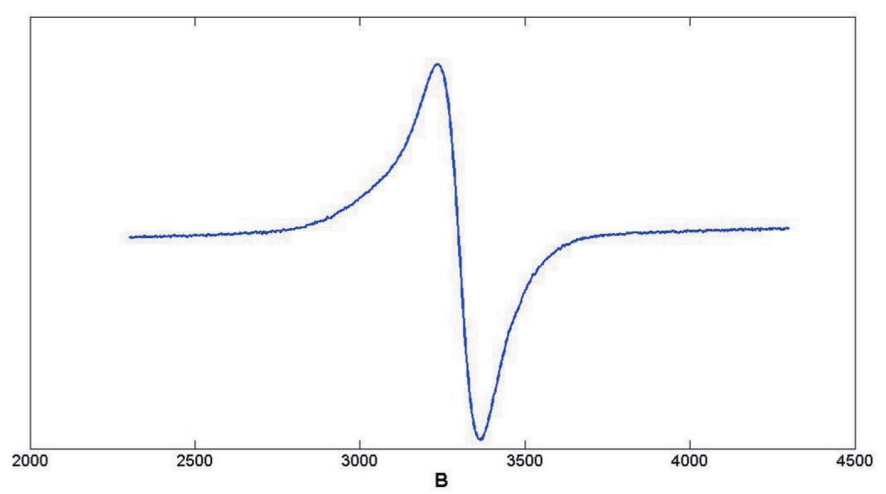

Figure 3

X-band EPR spectrum of $\mathrm{Mo}(\mathrm{tfd})_{2}(\mathrm{bdt})\left(\mathrm{Co}(\mathrm{CO})_{2}\right)_{3}$ in hexane at $298 \mathrm{~K} ; g$ $=2.010$. 


\section{Refinement}

Crystal data, data collection and structure refinement details are summarized in Table 2. $\mathrm{H}$ atoms were placed in calculated positions and included in a riding-motion approximation with $U_{\text {iso }}(\mathrm{H})=1.2 U_{\text {eq }}(\mathrm{C})$. The $\mathrm{F}$ atoms of the $-\mathrm{CF}_{3}$ groups containing $\mathrm{C} 7$ and $\mathrm{C} 8$ were refined as disordered over two sets of sites with ratios of refined occupancies of 0.703 (7):0.297 (7) and $0.72(2): 0.28(2)$, respectively.

\section{Acknowledgements}

We thank Professors Wolfang Kaim and Biprajit Sarkar (University of Stuttgart) for the use of their EPR spectrometer for the spectrum shown in Fig. 3.

\section{Funding information}

Funding for this research was provided by: Natural Sciences and Engineering Research Council of Canada ; University of Toronto.

\section{References}

Altomare, A., Cascarano, G., Giacovazzo, C., Guagliardi, A., Burla, M. C., Polidori, G. \& Camalli, M. (1994). J. Appl. Cryst. 27, 435.

Beswick, C. L., Schulman, J. M. \& Stiefel, E. I. (2004). Prog. Inorg. Chem. 52, 55-110.

Blessing, R. H. (1995). Acta Cryst. A51, 33-38.

Chen, Y.-Q., Zhang, J., Cai, S., Hou, X.-F., Schumann, H. \& Jin, G.-X. (2007). Dalton Trans. pp. 749-758.

Curtis, D. M., Druker, S. H., Goossen, L. \& Kampf, J. W. (1997). Organometallics, 16, 231-235.

Dickinson, R. G. \& Pauling, L. (1923). J. Am. Chem. Soc. 45, 14661471.

Farrugia, L. J. (2012). J. Appl. Cryst. 45, 849-854.

Groom, C. R., Bruno, I. J., Lightfoot, M. P. \& Ward, S. C. (2016). Acta Cryst. B72, 171-179.

Harrison, D. J., Lough, A. J., Nguyen, N. \& Fekl, U. (2007). Angew. Chem. Int. Ed. 46, 7644-7647.

Hinnemann, B., Moses, P. G. \& Nørskov, J. K. (2008). J. Phys. Condens. Matter, 20, 064236-064244.

Hosking, S., Lough, A. J. \& Fek1, U. (2009). Acta Cryst. E65, m759$\mathrm{m} 760$.

Murata, M., Habe, S., Araki, S., Namiki, K., Yamada, T., Nakagawa, N., Nankawa, T., Nihei, M., Mizutani, J., Kurihara, M. \& Nishihara, H. (2006). Inorg. Chem. 45, 1108-1116.
Table 2

Experimental details.

\begin{tabular}{|c|c|}
\hline \multicolumn{2}{|l|}{ Crystal data } \\
\hline Chemical formula & {$\left[\mathrm{Co}_{3} \mathrm{Mo}\left(\mathrm{C}_{4} \mathrm{~F}_{6} \mathrm{~S}_{2}\right)_{2}\left(\mathrm{C}_{6} \mathrm{H}_{4} \mathrm{~S}_{2}\right)(\mathrm{CO})_{6}\right]$} \\
\hline$M_{\mathrm{r}}$ & 1033.32 \\
\hline Crystal system, space group & Monoclinic, $C 2 / c$ \\
\hline Temperature (K) & 150 \\
\hline$a, b, c(\AA)$ & $\begin{array}{l}22.7465(5), 12.8779(5), \\
\quad 23.6033(6)\end{array}$ \\
\hline$\beta\left(^{\circ}\right)$ & $115.3840(16)$ \\
\hline$V\left(\AA^{3}\right)$ & $6246.5(3)$ \\
\hline$Z$ & 8 \\
\hline Radiation type & Мo $K \alpha$ \\
\hline$\mu\left(\mathrm{mm}^{-1}\right)$ & 2.47 \\
\hline Crystal size $(\mathrm{mm})$ & $0.32 \times 0.12 \times 0.10$ \\
\hline \multicolumn{2}{|l|}{ Data collection } \\
\hline Diffractometer & Nonius KappaCCD \\
\hline Absorption correction & $\begin{array}{l}\text { Multi-scan (SORTAV; Blessing, } \\
\text { 1995) }\end{array}$ \\
\hline$T_{\min }, T_{\max }$ & $0.686,0.798$ \\
\hline $\begin{array}{l}\text { No. of measured, independent and } \\
\text { observed }[I>2 \sigma(I)] \text { reflections }\end{array}$ & $21250,7102,5019$ \\
\hline$R_{\text {int }}$ & 0.046 \\
\hline$(\sin \theta / \lambda)_{\max }\left(\AA^{-1}\right)$ & 0.650 \\
\hline \multicolumn{2}{|l|}{ Refinement } \\
\hline$R\left[F^{2}>2 \sigma\left(F^{2}\right)\right], w R\left(F^{2}\right), S$ & $0.045,0.112,1.07$ \\
\hline No. of reflections & 7102 \\
\hline No. of parameters & 489 \\
\hline No. of restraints & 210 \\
\hline $\mathrm{H}$-atom treatment & H-atom parameters constrained \\
\hline$\Delta \rho_{\max }, \Delta \rho_{\min }\left(\mathrm{e} \AA^{-3}\right)$ & $1.09,-0.74$ \\
\hline
\end{tabular}

Computer programs: COLLECT (Nonius, 2002), DENZO-SMN (Otwinowski \& Minor, 1997), SIR92 (Altomare et al., 1994), SHELXL2018 (Sheldrick, 2015), PLATON (Spek, 2009) and SHELXTL (Sheldrick, 2008).

Muratsugu, S., Sodeyama, K., Kitamura, F., Tsukada, S., Tada, M., Tsuneyuki, S. \& Nishihara, H. (2011). Chem. Sci. 2, 1960-1968.

Nguyen, N., Harrison, D. J., Lough, A. J., De Crisci, A. G. \& Fekl, U. (2010). Eur. J. Inorg. Chem. pp. 3577-3585.

Nihei, M., Nankawa, T., Kurihara, M. \& Nishihara, H. (1999). Angew. Chem. Int. Ed. 38, 1098-1100.

Nonius, B. (2002). COLLECT. Nonius BV, Delft, The Netherlands.

Otwinowski, Z. \& Minor, W. (1997). Methods in Enzymology, Vol. 276, Macromolecular Crystallography, Part A edited by C. W. Carter \& R. M. Sweet pp. 307-326. London: Academic Press.

Sheldrick, G. M. (2008). Acta Cryst. A64, 112-122.

Sheldrick, G. M. (2015). Acta Cryst. C71, 3-8.

Spek, A. L. (2009). Acta Cryst. D65, 148-155. 


\section{supporting information}

Acta Cryst. (2019). E75, 1261-1264 [https://doi.org/10.1107/S2056989019010363]

\section{A molybdenum tris(dithiolene) complex coordinates to three bound cobalt centers in three different ways}

\section{Neilson Nguyen, Alan J. Lough and Ulrich Fekl}

Computing details

Data collection: COLLECT (Nonius, 2002); cell refinement: DENZO-SMN (Otwinowski \& Minor, 1997); data reduction: DENZO-SMN (Otwinowski \& Minor, 1997); program(s) used to solve structure: SIR92 (Altomare et al., 1994); program(s) used to refine structure: SHELXL2018 (Sheldrick, 2015); molecular graphics: PLATON (Spek, 2009); software used to prepare material for publication: SHELXTL (Sheldrick, 2008).

( $\mu_{3}$-Benzene-1,2-dithiolato) hexacarbonylbis $\left(\mu_{3}-1,1,1,4,4,4\right.$-hexafluorobut-2-ene-2,3dithiolato)tricobaltmolybdenum

Crystal data

$\left[\mathrm{Co}_{3} \mathrm{Mo}\left(\mathrm{C}_{4} \mathrm{~F}_{6} \mathrm{~S}_{2}\right)_{2}\left(\mathrm{C}_{6} \mathrm{H}_{4} \mathrm{~S}_{2}\right)(\mathrm{CO})_{6}\right]$

$F(000)=3992$

$M_{r}=1033.32$

Monoclinic, $C 2 / c$

$D_{\mathrm{x}}=2.198 \mathrm{Mg} \mathrm{m}^{-3}$

$a=22.7465(5) \AA$

$b=12.8779(5) \AA$

$c=23.6033(6) \AA$

$\beta=115.3840(16)^{\circ}$

$V=6246.5(3) \AA^{3}$

Mo $K \alpha$ radiation, $\lambda=0.71073 \AA$

Cell parameters from 21250 reflections

$\theta=3.0-27.5^{\circ}$

$\mu=2.47 \mathrm{~mm}^{-1}$

$T=150 \mathrm{~K}$

$Z=8$

Needle, dark red

Data collection

Nonius KappaCCD

$0.32 \times 0.12 \times 0.10 \mathrm{~mm}$

diffractometer

Radiation source: fine-focus sealed tube Detector resolution: 9 pixels $\mathrm{mm}^{-1}$ $\varphi$ scans and $\omega$ scans with $\kappa$ offsets Absorption correction: multi-scan (SORTAV; Blessing, 1995)

$T_{\min }=0.686, T_{\max }=0.798$

21250 measured reflections

7102 independent reflections 5019 reflections with $I>2 \sigma(I)$

$R_{\text {int }}=0.046$

$\theta_{\max }=27.5^{\circ}, \theta_{\min }=3.0^{\circ}$

$h=-29 \rightarrow 29$

$k=-16 \rightarrow 16$

$l=-30 \rightarrow 30$

\section{Refinement}

Refinement on $F^{2}$

Least-squares matrix: full

$R\left[F^{2}>2 \sigma\left(F^{2}\right)\right]=0.045$

$w R\left(F^{2}\right)=0.112$

$S=1.07$

7102 reflections

489 parameters

Primary atom site location: structure-invariant direct methods

Secondary atom site location: difference Fourier map

Hydrogen site location: inferred from neighbouring sites

210 restraints

$\mathrm{H}$-atom parameters constrained 
$w=1 /\left[\sigma^{2}\left(F_{\mathrm{o}}^{2}\right)+(0.054 P)^{2}+5.1797 P\right]$

where $P=\left(F_{\mathrm{o}}^{2}+2 F_{\mathrm{c}}^{2}\right) / 3$

$(\Delta / \sigma)_{\max }=0.001$

$$
\Delta \rho_{\max }=1.09 \mathrm{e} \AA^{-3}
$$

$\Delta \rho_{\min }=-0.74$ e $\AA^{-3}$

\section{Special details}

Geometry. All esds (except the esd in the dihedral angle between two 1.s. planes) are estimated using the full covariance matrix. The cell esds are taken into account individually in the estimation of esds in distances, angles and torsion angles; correlations between esds in cell parameters are only used when they are defined by crystal symmetry. An approximate (isotropic) treatment of cell esds is used for estimating esds involving 1.s. planes.

Fractional atomic coordinates and isotropic or equivalent isotropic displacement parameters $\left(\AA^{2}\right)$

\begin{tabular}{|c|c|c|c|c|c|}
\hline & $x$ & $y$ & $z$ & $U_{\text {iso }} * / U_{\text {eq }}$ & Occ. $(<1)$ \\
\hline Mo1 & $0.37011(2)$ & 0.67610 & $0.12477(2)$ & $0.02586(11)$ & \\
\hline Col & $0.32882(3)$ & $0.85549(5)$ & $0.15702(3)$ & $0.03548(16)$ & \\
\hline $\mathrm{Co} 2$ & $0.33267(3)$ & $0.47117(5)$ & $0.13309(3)$ & $0.03083(16)$ & \\
\hline $\mathrm{Co} 3$ & 0.45749 & $0.63936(5)$ & $0.08184(3)$ & $0.02962(15)$ & \\
\hline $\mathrm{S} 1$ & $0.32447(5)$ & $0.82941(9)$ & $0.06212(5)$ & $0.0297(2)$ & \\
\hline $\mathrm{S} 2$ & $0.36985(5)$ & $0.72385(9)$ & $0.22479(5)$ & $0.0331(3)$ & \\
\hline $\mathrm{S} 3$ & $0.26312(5)$ & $0.60667(9)$ & $0.10435(5)$ & $0.0296(2)$ & \\
\hline $\mathrm{S} 4$ & $0.45637(5)$ & $0.54398(9)$ & $0.16042(5)$ & $0.0301(2)$ & \\
\hline S5 & $0.35447(5)$ & $0.58534(9)$ & $0.02990(5)$ & $0.0295(2)$ & \\
\hline S6 & $0.47364(5)$ & $0.76648(9)$ & $0.15395(5)$ & $0.0307(3)$ & \\
\hline $\mathrm{F} 1$ & $0.26591(12)$ & $0.4072(2)$ & $-0.02994(12)$ & $0.0493(7)$ & \\
\hline $\mathrm{F} 2$ & $0.35969(14)$ & $0.3736(2)$ & $-0.02658(12)$ & $0.0484(7)$ & \\
\hline F3 & $0.32346(13)$ & $0.2830(2)$ & $0.02808(12)$ & $0.0456(7)$ & \\
\hline F4 & $0.41197(15)$ & $0.2522(2)$ & $0.14259(14)$ & $0.0581(8)$ & \\
\hline F5 & $0.46739(15)$ & $0.3033(2)$ & $0.09356(15)$ & $0.0588(8)$ & \\
\hline F6 & $0.49947(14)$ & $0.3377(2)$ & $0.19160(14)$ & $0.0635(9)$ & \\
\hline F7 & $0.2282(2)$ & $0.7313(7)$ & $0.2705(2)$ & $0.074(2)$ & $0.703(7)$ \\
\hline F8 & $0.3246(3)$ & $0.7860(5)$ & $0.3117(3)$ & $0.0698(18)$ & $0.703(7)$ \\
\hline F9 & $0.3064(4)$ & $0.6287(4)$ & $0.3225(2)$ & 0.0766 (19) & $0.703(7)$ \\
\hline F7A & $0.2573(8)$ & $0.7953(11)$ & $0.2754(6)$ & $0.072(4)$ & $0.297(7)$ \\
\hline F8A & $0.3435(5)$ & $0.7165(15)$ & $0.3305(4)$ & $0.063(3)$ & $0.297(7)$ \\
\hline F9A & $0.2537(7)$ & $0.6372(10)$ & $0.2967(5)$ & $0.063(3)$ & $0.297(7)$ \\
\hline F10 & $0.1632(4)$ & $0.5120(8)$ & $0.1246(4)$ & $0.055(2)$ & $0.72(2)$ \\
\hline F11 & $0.1431(3)$ & $0.6580(6)$ & $0.1565(6)$ & $0.066(2)$ & $0.72(2)$ \\
\hline F12 & $0.1944(4)$ & $0.5381(9)$ & $0.2222(3)$ & $0.068(2)$ & $0.72(2)$ \\
\hline F10A & $0.1475(9)$ & $0.558(2)$ & $0.1103(7)$ & $0.057(4)$ & $0.28(2)$ \\
\hline F11A & $0.1543(10)$ & $0.6471(14)$ & $0.1877(12)$ & $0.056(4)$ & $0.28(2)$ \\
\hline F12A & $0.1972(10)$ & $0.4990(14)$ & $0.2037(10)$ & $0.057(4)$ & $0.28(2)$ \\
\hline $\mathrm{O} 1$ & $0.3951(3)$ & $1.0559(4)$ & 0.2075 & $0.112(2)$ & \\
\hline $\mathrm{O} 2$ & $0.19536(19)$ & $0.9067(4)$ & $0.1404(2)$ & $0.0771(13)$ & \\
\hline $\mathrm{O} 3$ & $0.37570(16)$ & 0.4528 & $0.26994(14)$ & $0.0486(9)$ & \\
\hline $\mathrm{O} 4$ & $0.23791(19)$ & $0.2999(3)$ & $0.09880(16)$ & $0.0587(11)$ & \\
\hline $\mathrm{O} 5$ & $0.56008(17)$ & $0.5074(3)$ & $0.07720(17)$ & $0.0586(10)$ & \\
\hline O6 & $0.4473(2)$ & $0.7725(3)$ & $-0.02293(17)$ & $0.0610(10)$ & \\
\hline $\mathrm{C} 1$ & $0.35680(19)$ & $0.4568(3)$ & $0.06092(19)$ & $0.0304(10)$ & \\
\hline $\mathrm{C} 2$ & $0.41017(19)$ & $0.4338(4)$ & $0.1204(2)$ & $0.0323(10)$ & \\
\hline
\end{tabular}




$\begin{array}{lllll}\text { C3 } & 0.3269(2) & 0.3798(4) & 0.0090(2) & 0.0372(11) \\ \text { C4 } & 0.4467(2) & 0.3306(4) & 0.1367(2) & 0.0423(12) \\ \text { C5 } & 0.29505(19) & 0.6793(4) & 0.22259(18) & 0.0309(10) \\ \text { C6 } & 0.25188(19) & 0.6280(3) & 0.17312(19) & 0.0304(10) \\ \text { C7 } & 0.2880(2) & 0.7059(4) & 0.2817(2) & 0.0437(12) \\ \text { C8 } & 0.1880(2) & 0.5842(4) & 0.1692(2) & 0.0435(12) \\ \text { C9 } & 0.3691(3) & 0.9805(5) & 0.1880(3) & 0.0646(17) \\ \text { C10 } & 0.2465(3) & 0.8898(4) & 0.1474(2) & 0.0495(13) \\ \text { C11 } & 0.3593(2) & 0.4622(4) & 0.2178(2) & 0.0354(10) \\ \text { C12 } & 0.2757(2) & 0.3625(4) & 0.1124(2) & 0.0409(11) \\ \text { C13 } & 0.5214(2) & 0.5607(4) & 0.0791(2) & 0.0370(11) \\ \text { C14 } & 0.4513(2) & 0.7229(4) & 0.0182(2) & 0.0410(11) \\ \text { C15 } & 0.45531(19) & 0.8839(3) & 0.11004(19) & 0.0296(9) \\ \text { C16 } & 0.5057(2) & 0.9503(4) & 0.1170(2) & 0.0398(11) \\ \text { H16A } & 0.549037 & 0.934569 & 0.146045 & 0.048^{*} \\ \text { C17 } & 0.4928(2) & 1.0394(4) & 0.0814(2) & 0.0512(14) \\ \text { H17A } & 0.527525 & 1.083894 & 0.085070 & 0.061^{*} \\ \text { C18 } & 0.4294(2) & 1.0649(4) & 0.0401(2) & 0.0442(12) \\ \text { H18A } & 0.420821 & 1.127055 & 0.016168 & 0.053^{*} \\ \text { C19 } & 0.3788(2) & 0.9995(4) & 0.0339(2) & 0.0347(10) \\ \text { H19A } & 0.335466 & 1.016576 & 0.005600 & 0.042^{*} \\ \text { C20 } & 0.39134(19) & 0.9089(3) & 0.06908(19) & 0.0291(9)\end{array}$

Atomic displacement parameters $\left(\AA^{2}\right)$

\begin{tabular}{lllllll}
\hline & $U^{11}$ & $U^{22}$ & $U^{33}$ & $U^{12}$ & $U^{13}$ & $U^{23}$ \\
\hline Mo1 & $0.02129(18)$ & $0.0321(2)$ & $0.02433(19)$ & $-0.00421(15)$ & $0.00990(15)$ & $-0.00087(15)$ \\
Co1 & $0.0354(3)$ & $0.0368(4)$ & $0.0387(3)$ & $-0.0013(3)$ & $0.0202(3)$ & $-0.0033(3)$ \\
Co2 & $0.0325(3)$ & $0.0345(4)$ & $0.0287(3)$ & $-0.0072(3)$ & $0.0162(3)$ & $-0.0013(2)$ \\
Co3 & $0.0259(3)$ & $0.0351(4)$ & $0.0308(3)$ & $-0.0016(3)$ & $0.0149(2)$ & $0.0027(3)$ \\
S1 & $0.0216(5)$ & $0.0358(6)$ & $0.0311(5)$ & $-0.0030(4)$ & $0.0107(4)$ & $0.0019(5)$ \\
S2 & $0.0278(5)$ & $0.0441(7)$ & $0.0269(5)$ & $-0.0067(5)$ & $0.0112(4)$ & $-0.0048(5)$ \\
S3 & $0.0242(5)$ & $0.0394(7)$ & $0.0261(5)$ & $-0.0077(5)$ & $0.0117(4)$ & $-0.0025(5)$ \\
S4 & $0.0286(5)$ & $0.0344(6)$ & $0.0283(5)$ & $-0.0017(5)$ & $0.0130(4)$ & $0.0029(5)$ \\
S5 & $0.0275(5)$ & $0.0357(6)$ & $0.0269(5)$ & $-0.0036(5)$ & $0.0133(4)$ & $-0.0001(5)$ \\
S6 & $0.0229(5)$ & $0.0362(7)$ & $0.0304(5)$ & $-0.0045(4)$ & $0.0088(4)$ & $0.0008(5)$ \\
F1 & $0.0445(15)$ & $0.0509(18)$ & $0.0423(15)$ & $-0.0106(14)$ & $0.0088(13)$ & $-0.0117(13)$ \\
F2 & $0.0626(17)$ & $0.0540(19)$ & $0.0453(15)$ & $-0.0151(15)$ & $0.0391(14)$ & $-0.0152(13)$ \\
F3 & $0.0604(17)$ & $0.0358(16)$ & $0.0460(15)$ & $-0.0124(13)$ & $0.0281(13)$ & $-0.0079(12)$ \\
F4 & $0.0690(19)$ & $0.0357(17)$ & $0.074(2)$ & $-0.0050(15)$ & $0.0343(17)$ & $0.0094(15)$ \\
F5 & $0.073(2)$ & $0.0481(19)$ & $0.072(2)$ & $0.0172(16)$ & $0.0474(18)$ & $0.0034(15)$ \\
F6 & $0.0556(19)$ & $0.0473(19)$ & $0.0605(19)$ & $0.0085(15)$ & $-0.0008(16)$ & $0.0074(15)$ \\
F7 & $0.043(3)$ & $0.130(6)$ & $0.055(3)$ & $0.008(3)$ & $0.027(2)$ & $-0.032(4)$ \\
F8 & $0.092(4)$ & $0.085(4)$ & $0.054(3)$ & $-0.038(4)$ & $0.051(3)$ & $-0.038(3)$ \\
F9 & $0.124(5)$ & $0.081(4)$ & $0.033(3)$ & $0.019(4)$ & $0.041(3)$ & $0.012(3)$ \\
F7A & $0.097(8)$ & $0.077(8)$ & $0.052(6)$ & $0.022(7)$ & $0.042(6)$ & $-0.017(6)$ \\
F8A & $0.061(6)$ & $0.106(9)$ & $0.028(5)$ & $-0.005(7)$ & $0.025(5)$ & $-0.006(6)$ \\
F9A & $0.083(8)$ & $0.081(7)$ & $0.038(6)$ & $-0.025(7)$ & $0.038(5)$ & $-0.011(5)$
\end{tabular}




\begin{tabular}{|c|c|c|c|c|c|c|}
\hline F10 & 0.045 & $0.069(5)$ & $0.063(4)$ & $-0.029(3)$ & $0.035(3)$ & $-0.024(3)$ \\
\hline F11 & $0.030(3)$ & $0.084(4)$ & $0.084(5)$ & $0.006(2)$ & $0.024(3)$ & $-0.006(4)$ \\
\hline F12 & $0.062(3)$ & $0.099(5)$ & $0.053(3)$ & $-0.032(4)$ & $0.035(3)$ & 0.005 (4) \\
\hline F10A & $0.031(6)$ & $0.087(10)$ & $0.052(7)$ & $-0.023(7)$ & $0.017(5)$ & $-0.012(7)$ \\
\hline F11A & $0.044(7)$ & $0.067(7)$ & $0.072(9)$ & $-0.008(6)$ & $0.039(7)$ & $-0.017(7)$ \\
\hline F12A & $0.055(6)$ & $0.065(8)$ & $0.063(8)$ & $-0.014(7)$ & $0.036(6)$ & $0.005(7)$ \\
\hline $\mathrm{O} 1$ & $0.183(5)$ & $0.076(4)$ & $0.116(4)$ & $-0.071(4)$ & $0.102(4)$ & $-0.047(3)$ \\
\hline $\mathrm{O} 2$ & $0.058(2)$ & $0.107(4)$ & $0.082(3)$ & $0.038(3)$ & $0.045(2)$ & $0.027(3)$ \\
\hline $\mathrm{O} 3$ & $0.053(2)$ & $0.063(3)$ & $0.0311(18)$ & $0.0045(18)$ & $0.0197(16)$ & $0.0060(16)$ \\
\hline $\mathrm{O} 4$ & $0.078(3)$ & $0.063(3)$ & $0.051(2)$ & $-0.042(2)$ & $0.043(2)$ & $-0.0207(19)$ \\
\hline O5 & $0.052(2)$ & $0.068(3)$ & $0.069(2)$ & $0.020(2)$ & $0.0381(19)$ & $0.012(2)$ \\
\hline O6 & $0.091(3)$ & $0.053(2)$ & $0.054(2)$ & $-0.002(2)$ & $0.045(2)$ & $0.0113(19)$ \\
\hline $\mathrm{C} 1$ & $0.031(2)$ & $0.030(2)$ & $0.034(2)$ & $-0.0034(19)$ & $0.0177(19)$ & $-0.0025(19)$ \\
\hline $\mathrm{C} 2$ & $0.033(2)$ & $0.034(3)$ & $0.035(2)$ & $-0.0041(19)$ & $0.0195(19)$ & $-0.0018(19)$ \\
\hline $\mathrm{C} 3$ & $0.039(3)$ & $0.041(3)$ & $0.037(2)$ & $-0.007(2)$ & $0.022(2)$ & $-0.003(2)$ \\
\hline $\mathrm{C} 4$ & $0.048(3)$ & $0.032(3)$ & $0.047(3)$ & $-0.007(2)$ & $0.021(2)$ & $0.000(2)$ \\
\hline $\mathrm{C} 5$ & $0.027(2)$ & $0.039(3)$ & $0.028(2)$ & $-0.0028(19)$ & $0.0126(18)$ & $0.0002(19)$ \\
\hline C6 & $0.027(2)$ & $0.036(3)$ & $0.031(2)$ & $0.0008(19)$ & $0.0146(19)$ & $0.0010(19)$ \\
\hline $\mathrm{C} 7$ & $0.044(3)$ & $0.061(3)$ & $0.032(2)$ & $-0.001(3)$ & $0.022(2)$ & $-0.006(2)$ \\
\hline $\mathrm{C} 8$ & $0.036(2)$ & $0.059(3)$ & $0.044(3)$ & $-0.010(2)$ & $0.024(2)$ & $-0.002(2)$ \\
\hline C9 & $0.098(5)$ & $0.056(4)$ & $0.065(4)$ & $-0.029(4)$ & $0.059(4)$ & $-0.022(3)$ \\
\hline $\mathrm{C} 10$ & $0.058(3)$ & $0.049(3)$ & $0.052(3)$ & $0.010(3)$ & $0.034(3)$ & $0.003(3)$ \\
\hline C11 & $0.036(2)$ & $0.034(3)$ & $0.039(3)$ & $-0.001(2)$ & $0.019(2)$ & $0.000(2)$ \\
\hline $\mathrm{C} 12$ & $0.053(3)$ & $0.046(3)$ & $0.032(2)$ & -0.009 (3) & $0.026(2)$ & $-0.008(2)$ \\
\hline $\mathrm{C} 13$ & $0.034(2)$ & $0.042(3)$ & 0.040 & $0.003(2)$ & $0.020(2)$ & $0.009(2)$ \\
\hline $\mathrm{C} 14$ & $0.039(3)$ & $0.045(3)$ & $0.045(3)$ & $-0.001(2)$ & $0.024(2)$ & $-0.005(2)$ \\
\hline $\mathrm{C} 15$ & $0.029(2)$ & $0.032(3)$ & $0.030(2)$ & $-0.0031(18)$ & $0.0144(19)$ & $-0.0032(18)$ \\
\hline $\mathrm{C} 16$ & $0.026(2)$ & $0.040(3)$ & $0.048(3)$ & $-0.008(2)$ & $0.011(2)$ & $0.003(2)$ \\
\hline $\mathrm{C} 17$ & $0.045(3)$ & $0.042(3)$ & $0.064(3)$ & $-0.017(2)$ & $0.021(3)$ & $0.005(3)$ \\
\hline $\mathrm{C} 18$ & $0.043(3)$ & 0.035 & 0.050 & $-0.004(2)$ & $0.016(2)$ & 0.009 (2) \\
\hline C19 & $0.029(2)$ & $0.036(3)$ & $0.037(2)$ & $-0.002(2)$ & $0.012(2)$ & $-0.003(2)$ \\
\hline $\mathrm{C} 20$ & $0.026(2)$ & $0.034(3)$ & $0.029(2)$ & $-0.0074(18)$ & $0.0129(18)$ & $-0.0057(19)$ \\
\hline
\end{tabular}

Geometric parameters $\left(\stackrel{A}{\circ}{ }^{\circ}\right)$

\begin{tabular}{llll}
\hline Mo1-S5 & $2.4134(11)$ & F6-C4 & $1.339(5)$ \\
Mo1-S1 & $2.4179(11)$ & F7-C7 & $1.312(6)$ \\
Mo1-S3 & $2.4391(10)$ & F8-C7 & $1.324(6)$ \\
Mo1-S2 & $2.4420(11)$ & F9-C7 & $1.321(6)$ \\
Mo1-S6 & $2.4461(11)$ & F7A-C7 & $1.322(10)$ \\
Mo1-S4 & $2.4571(11)$ & F8A-C7 & $1.300(10)$ \\
Mo1-Co3 & $2.6320(6)$ & F9A-C7 & $1.324(9)$ \\
Mo1-Co1 & $2.7224(7)$ & F10-C8 & $1.333(7)$ \\
Mo1-Co2 & $2.8058(7)$ & F11-C8 & $1.332(7)$ \\
Co1-C10 & $1.840(5)$ & F12-C8 & $1.336(7)$ \\
Co1-C9 & $1.842(6)$ & F10A-C8 & $1.341(12)$ \\
Co1-S1 & $2.2247(12)$ & F11A-C8 & $1.312(12)$ \\
Co1-S2 & $2.2414(13)$ & F12A-C8 & $1.329(12)$
\end{tabular}




\begin{tabular}{|c|c|c|c|}
\hline $\mathrm{Co} 2-\mathrm{C} 12$ & $1.825(5)$ & $\mathrm{O} 1-\mathrm{C} 9$ & $1.127(7)$ \\
\hline $\mathrm{Co} 2-\mathrm{C} 11$ & $1.827(5)$ & $\mathrm{O} 2-\mathrm{C} 10$ & $1.125(6)$ \\
\hline $\mathrm{Co} 2-\mathrm{C} 2$ & $1.970(4)$ & $\mathrm{O} 3-\mathrm{C} 11$ & $1.130(5)$ \\
\hline $\mathrm{Co} 2-\mathrm{C} 1$ & $2.010(4)$ & $\mathrm{O} 4-\mathrm{C} 12$ & $1.122(5)$ \\
\hline $\mathrm{Co} 2-\mathrm{S} 3$ & $2.2556(13)$ & $\mathrm{O} 5-\mathrm{C} 13$ & $1.131(5)$ \\
\hline $\mathrm{Co} 2-\mathrm{S} 4$ & $2.7671(11)$ & $\mathrm{O} 6-\mathrm{C} 14$ & $1.133(6)$ \\
\hline $\mathrm{Co} 3-\mathrm{C} 13$ & $1.796(5)$ & $\mathrm{C} 1-\mathrm{C} 2$ & $1.439(6)$ \\
\hline $\mathrm{Co} 3-\mathrm{C} 14$ & $1.803(5)$ & $\mathrm{C} 1-\mathrm{C} 3$ & $1.494(6)$ \\
\hline $\mathrm{Co} 3-\mathrm{S} 4$ & $2.2338(12)$ & $\mathrm{C} 2-\mathrm{C} 4$ & $1.528(7)$ \\
\hline $\mathrm{Co} 3-\mathrm{S} 5$ & $2.2395(11)$ & $\mathrm{C} 5-\mathrm{C} 6$ & $1.335(6)$ \\
\hline $\mathrm{Co} 3-\mathrm{S} 6$ & $2.2749(13)$ & $\mathrm{C} 5-\mathrm{C} 7$ & $1.509(6)$ \\
\hline $\mathrm{S} 1-\mathrm{C} 20$ & $1.782(4)$ & $\mathrm{C} 6-\mathrm{C} 8$ & $1.524(6)$ \\
\hline $\mathrm{S} 2-\mathrm{C} 5$ & $1.775(4)$ & $\mathrm{C} 15-\mathrm{C} 16$ & $1.383(6)$ \\
\hline $\mathrm{S} 3-\mathrm{C} 6$ & $1.770(4)$ & $\mathrm{C} 15-\mathrm{C} 20$ & $1.396(6)$ \\
\hline $\mathrm{S} 4-\mathrm{C} 2$ & $1.777(4)$ & $\mathrm{C} 16-\mathrm{C} 17$ & $1.377(7)$ \\
\hline $\mathrm{S} 5-\mathrm{C} 1$ & $1.801(4)$ & $\mathrm{C} 16-\mathrm{H} 16 \mathrm{~A}$ & 0.9500 \\
\hline $\mathrm{S} 6-\mathrm{C} 15$ & $1.779(4)$ & $\mathrm{C} 17-\mathrm{C} 18$ & $1.390(7)$ \\
\hline $\mathrm{F} 1-\mathrm{C} 3$ & $1.341(5)$ & C17-H17A & 0.9500 \\
\hline $\mathrm{F} 2-\mathrm{C} 3$ & $1.344(5)$ & $\mathrm{C} 18-\mathrm{C} 19$ & $1.381(6)$ \\
\hline $\mathrm{F} 3-\mathrm{C} 3$ & $1.339(5)$ & $\mathrm{C} 18-\mathrm{H} 18 \mathrm{~A}$ & 0.9500 \\
\hline $\mathrm{F} 4-\mathrm{C} 4$ & $1.324(5)$ & $\mathrm{C} 19-\mathrm{C} 20$ & $1.389(6)$ \\
\hline $\mathrm{F} 5-\mathrm{C} 4$ & $1.340(6)$ & C19-H19A & 0.9500 \\
\hline $\mathrm{S} 5-\mathrm{Mo} 1-\mathrm{S} 1$ & $88.52(4)$ & $\mathrm{C} 2-\mathrm{S} 4-\mathrm{Co} 3$ & $102.26(15)$ \\
\hline $\mathrm{S} 5-\mathrm{Mo1}-\mathrm{S} 3$ & $84.34(4)$ & $\mathrm{C} 2-\mathrm{S} 4-\mathrm{Mo} 1$ & $99.62(14)$ \\
\hline $\mathrm{S} 1-\mathrm{Mo1}-\mathrm{S} 3$ & $92.75(4)$ & $\mathrm{Co} 3-\mathrm{S} 4-\mathrm{Mo} 1$ & $68.07(3)$ \\
\hline $\mathrm{S} 5-\mathrm{Mo} 1-\mathrm{S} 2$ & $163.43(4)$ & $\mathrm{C} 2-\mathrm{S} 4-\mathrm{Co} 2$ & $45.16(13)$ \\
\hline $\mathrm{S} 1-\mathrm{Mo} 1-\mathrm{S} 2$ & $101.96(4)$ & $\mathrm{Co} 3-\mathrm{S} 4-\mathrm{Co} 2$ & $111.42(4)$ \\
\hline $\mathrm{S} 3-\mathrm{Mo} 1-\mathrm{S} 2$ & $82.39(4)$ & $\mathrm{Mo} 1-\mathrm{S} 4-\mathrm{Co} 2$ & $64.65(3)$ \\
\hline $\mathrm{S} 5-\mathrm{Mo1}-\mathrm{S} 6$ & $103.47(4)$ & $\mathrm{C} 1-\mathrm{S} 5-\mathrm{Co} 3$ & $102.78(13)$ \\
\hline $\mathrm{S} 1-\mathrm{Mo1}-\mathrm{S} 6$ & $83.85(4)$ & $\mathrm{C} 1-\mathrm{S} 5-\mathrm{Mo} 1$ & $95.79(14)$ \\
\hline $\mathrm{S} 3-\mathrm{Mo1}-\mathrm{S} 6$ & $171.36(4)$ & $\mathrm{Co} 3-\mathrm{S} 5-\mathrm{Mo} 1$ & $68.78(3)$ \\
\hline $\mathrm{S} 2-\mathrm{Mo} 1-\mathrm{S} 6$ & $90.52(4)$ & $\mathrm{C} 15-\mathrm{S} 6-\mathrm{Co} 3$ & $104.69(14)$ \\
\hline $\mathrm{S} 5-\mathrm{Mo1}-\mathrm{S} 4$ & 76.17 (4) & $\mathrm{C} 15-\mathrm{S} 6-\mathrm{Mo} 1$ & $106.48(13)$ \\
\hline $\mathrm{S} 1-\mathrm{Mo1}-\mathrm{S} 4$ & $147.71(4)$ & $\mathrm{Co} 3-\mathrm{S} 6-\mathrm{Mo} 1$ & $67.65(3)$ \\
\hline $\mathrm{S} 3-\mathrm{Mo1}-\mathrm{S} 4$ & $113.36(4)$ & $\mathrm{C} 2-\mathrm{C} 1-\mathrm{C} 3$ & $124.1(4)$ \\
\hline $\mathrm{S} 2-\mathrm{Mo1}-\mathrm{S} 4$ & $100.13(4)$ & $\mathrm{C} 2-\mathrm{C} 1-\mathrm{S} 5$ & $116.8(3)$ \\
\hline $\mathrm{S} 6-\mathrm{Mo1}-\mathrm{S} 4$ & $72.66(4)$ & $\mathrm{C} 3-\mathrm{C} 1-\mathrm{S} 5$ & $110.6(3)$ \\
\hline $\mathrm{S} 5-\mathrm{Mo} 1-\mathrm{Co} 3$ & $52.48(3)$ & $\mathrm{C} 2-\mathrm{C} 1-\mathrm{Co} 2$ & $67.3(2)$ \\
\hline $\mathrm{S} 1-\mathrm{Mo} 1-\mathrm{Co} 3$ & $96.23(3)$ & $\mathrm{C} 3-\mathrm{C} 1-\mathrm{Co} 2$ & $124.2(3)$ \\
\hline $\mathrm{S} 3-\mathrm{Mo} 1-\mathrm{Co} 3$ & $135.41(3)$ & $\mathrm{S} 5-\mathrm{C} 1-\mathrm{Co} 2$ & $106.8(2)$ \\
\hline $\mathrm{S} 2-\mathrm{Mo1}-\mathrm{Co} 3$ & $137.11(3)$ & $\mathrm{C} 1-\mathrm{C} 2-\mathrm{C} 4$ & $124.4(4)$ \\
\hline $\mathrm{S} 6-\mathrm{Mo1}-\mathrm{Co} 3$ & $53.08(3)$ & $\mathrm{C} 1-\mathrm{C} 2-\mathrm{S} 4$ & $114.4(3)$ \\
\hline $\mathrm{S} 4-\mathrm{Mo} 1-\mathrm{Co} 3$ & $51.93(3)$ & $\mathrm{C} 4-\mathrm{C} 2-\mathrm{S} 4$ & $115.3(3)$ \\
\hline $\mathrm{S} 5-\mathrm{Mo} 1-\mathrm{Co} 1$ & $137.65(3)$ & $\mathrm{C} 1-\mathrm{C} 2-\mathrm{Co} 2$ & $70.3(2)$ \\
\hline $\mathrm{S} 1-\mathrm{Mo1}-\mathrm{Co} 1$ & $50.87(3)$ & $\mathrm{C} 4-\mathrm{C} 2-\mathrm{Co} 2$ & $126.8(3)$ \\
\hline $\mathrm{S} 3-\mathrm{Mo1}-\mathrm{Co} 1$ & $86.08(3)$ & $\mathrm{S} 4-\mathrm{C} 2-\mathrm{Co} 2$ & $95.1(2)$ \\
\hline $\mathrm{S} 2-\mathrm{Mo} 1-\mathrm{Co} 1$ & $51.09(3)$ & $\mathrm{F} 3-\mathrm{C} 3-\mathrm{F} 1$ & $106.5(3)$ \\
\hline
\end{tabular}




\begin{tabular}{|c|c|c|c|}
\hline $\mathrm{S} 6-\mathrm{Mo} 1-\mathrm{Co} 1$ & $85.61(3)$ & $\mathrm{F} 3-\mathrm{C} 3-\mathrm{F} 2$ & $106.4(4)$ \\
\hline $\mathrm{S} 4-\mathrm{Mo1}-\mathrm{Co} 1$ & $144.34(3)$ & $\mathrm{F} 1-\mathrm{C} 3-\mathrm{F} 2$ & $106.0(3)$ \\
\hline $\mathrm{Co} 3-\mathrm{Mo} 1-\mathrm{Co} 1$ & $131.93(2)$ & $\mathrm{F} 3-\mathrm{C} 3-\mathrm{C} 1$ & $114.4(4)$ \\
\hline $\mathrm{S} 5-\mathrm{Mo} 1-\mathrm{Co} 2$ & $71.40(3)$ & $\mathrm{F} 1-\mathrm{C} 3-\mathrm{C} 1$ & $111.2(4)$ \\
\hline $\mathrm{S} 1-\mathrm{Mo} 1-\mathrm{Co} 2$ & $138.35(3)$ & $\mathrm{F} 2-\mathrm{C} 3-\mathrm{C} 1$ & $111.9(4)$ \\
\hline $\mathrm{S} 3-\mathrm{Mo} 1-\mathrm{Co} 2$ & $50.35(3)$ & $\mathrm{F} 4-\mathrm{C} 4-\mathrm{F} 6$ & $106.2(4)$ \\
\hline $\mathrm{S} 2-\mathrm{Mo} 1-\mathrm{Co} 2$ & $92.44(3)$ & $\mathrm{F} 4-\mathrm{C} 4-\mathrm{F} 5$ & $107.4(4)$ \\
\hline $\mathrm{S} 6-\mathrm{Mo} 1-\mathrm{Co} 2$ & $135.42(3)$ & $\mathrm{F} 6-\mathrm{C} 4-\mathrm{F} 5$ & $107.0(4)$ \\
\hline $\mathrm{S} 4-\mathrm{Mo} 1-\mathrm{Co} 2$ & $63.03(3)$ & $\mathrm{F} 4-\mathrm{C} 4-\mathrm{C} 2$ & $113.9(4)$ \\
\hline $\mathrm{Co} 3-\mathrm{Mo} 1-\mathrm{Co} 2$ & $99.20(2)$ & $\mathrm{F} 6-\mathrm{C} 4-\mathrm{C} 2$ & $110.4(4)$ \\
\hline $\mathrm{Co} 1-\mathrm{Mo} 1-\mathrm{Co} 2$ & $128.84(2)$ & $\mathrm{F} 5-\mathrm{C} 4-\mathrm{C} 2$ & $111.5(4)$ \\
\hline $\mathrm{C} 10-\mathrm{Co} 1-\mathrm{C} 9$ & $98.4(3)$ & $\mathrm{C} 6-\mathrm{C} 5-\mathrm{C} 7$ & $126.2(4)$ \\
\hline $\mathrm{C} 10-\mathrm{Co} 1-\mathrm{S} 1$ & $107.95(16)$ & $\mathrm{C} 6-\mathrm{C} 5-\mathrm{S} 2$ & $121.5(3)$ \\
\hline C9-Co1-S1 & $109.32(18)$ & $\mathrm{C} 7-\mathrm{C} 5-\mathrm{S} 2$ & $112.3(3)$ \\
\hline $\mathrm{C} 10-\mathrm{Co} 1-\mathrm{S} 2$ & $111.11(17)$ & $\mathrm{C} 5-\mathrm{C} 6-\mathrm{C} 8$ & $124.2(4)$ \\
\hline $\mathrm{C} 9-\mathrm{Co} 1-\mathrm{S} 2$ & $113.2(2)$ & $\mathrm{C} 5-\mathrm{C} 6-\mathrm{S} 3$ & $122.6(3)$ \\
\hline $\mathrm{S} 1-\mathrm{Co} 1-\mathrm{S} 2$ & $115.44(5)$ & $\mathrm{C} 8-\mathrm{C} 6-\mathrm{S} 3$ & $113.3(3)$ \\
\hline $\mathrm{C} 10-\mathrm{Co} 1-\mathrm{Mo} 1$ & $128.61(18)$ & $\mathrm{F} 7-\mathrm{C} 7-\mathrm{F} 9$ & $108.1(5)$ \\
\hline $\mathrm{C} 9-\mathrm{Co} 1-\mathrm{Mo1}$ & $132.8(2)$ & $\mathrm{F} 8 \mathrm{~A}-\mathrm{C} 7-\mathrm{F} 7 \mathrm{~A}$ & $105.8(10)$ \\
\hline $\mathrm{S} 1-\mathrm{Co} 1-\mathrm{Mo} 1$ & $57.46(3)$ & $\mathrm{F} 7-\mathrm{C} 7-\mathrm{F} 8$ & $105.8(5)$ \\
\hline $\mathrm{S} 2-\mathrm{Co} 1-\mathrm{Mo} 1$ & $57.97(3)$ & $\mathrm{F} 9-\mathrm{C} 7-\mathrm{F} 8$ & $105.4(5)$ \\
\hline $\mathrm{C} 12-\mathrm{Co} 2-\mathrm{C} 11$ & $96.3(2)$ & $\mathrm{F} 8 \mathrm{~A}-\mathrm{C} 7-\mathrm{F} 9 \mathrm{~A}$ & $107.0(9)$ \\
\hline $\mathrm{C} 12-\mathrm{Co} 2-\mathrm{C} 2$ & $110.5(2)$ & $\mathrm{F} 7 \mathrm{~A}-\mathrm{C} 7-\mathrm{F} 9 \mathrm{~A}$ & $105.6(10)$ \\
\hline $\mathrm{C} 11-\mathrm{Co} 2-\mathrm{C} 2$ & $104.60(18)$ & $\mathrm{F} 8 \mathrm{~A}-\mathrm{C} 7-\mathrm{C} 5$ & $113.3(6)$ \\
\hline $\mathrm{C} 12-\mathrm{Co} 2-\mathrm{C} 1$ & $97.04(18)$ & F7-C7-C5 & 112.5 \\
\hline $\mathrm{C} 11-\mathrm{Co} 2-\mathrm{C} 1$ & $146.99(18)$ & $\mathrm{F} 9-\mathrm{C} 7-\mathrm{C} 5$ & $112.2(4)$ \\
\hline $\mathrm{C} 2-\mathrm{Co} 2-\mathrm{C} 1$ & $42.40(16)$ & $\mathrm{F} 7 \mathrm{~A}-\mathrm{C} 7-\mathrm{C} 5$ & $110.7(6)$ \\
\hline $\mathrm{C} 12-\mathrm{Co} 2-\mathrm{S} 3$ & $100.76(17)$ & $\mathrm{F} 8-\mathrm{C} 7-\mathrm{C} 5$ & $112.3(4)$ \\
\hline $\mathrm{C} 11-\mathrm{Co} 2-\mathrm{S} 3$ & $103.81(15)$ & $\mathrm{F} 9 \mathrm{~A}-\mathrm{C} 7-\mathrm{C} 5$ & $113.8(6)$ \\
\hline $\mathrm{C} 2-\mathrm{Co} 2-\mathrm{S} 3$ & $134.51(14)$ & $\mathrm{F} 11 \mathrm{~A}-\mathrm{C} 8-\mathrm{F} 12 \mathrm{~A}$ & $105.5(11)$ \\
\hline $\mathrm{C} 1-\mathrm{Co} 2-\mathrm{S} 3$ & $103.14(13)$ & $\mathrm{F} 11-\mathrm{C} 8-\mathrm{F} 10$ & $107.2(5)$ \\
\hline $\mathrm{C} 12-\mathrm{Co} 2-\mathrm{S} 4$ & $148.89(16)$ & $\mathrm{F} 11-\mathrm{C} 8-\mathrm{F} 12$ & $107.0(6)$ \\
\hline $\mathrm{C} 11-\mathrm{Co} 2-\mathrm{S} 4$ & $86.51(14)$ & $\mathrm{F} 10-\mathrm{C} 8-\mathrm{F} 12$ & $105.6(6)$ \\
\hline $\mathrm{C} 2-\mathrm{Co} 2-\mathrm{S} 4$ & $39.76(13)$ & $\mathrm{F} 11 \mathrm{~A}-\mathrm{C} 8-\mathrm{F} 10 \mathrm{~A}$ & $105.2(11)$ \\
\hline $\mathrm{C} 1-\mathrm{Co} 2-\mathrm{S} 4$ & $66.97(12)$ & $\mathrm{F} 12 \mathrm{~A}-\mathrm{C} 8-\mathrm{F} 10 \mathrm{~A}$ & $106.5(11)$ \\
\hline $\mathrm{S} 3-\mathrm{Co} 2-\mathrm{S} 4$ & $108.66(4)$ & $\mathrm{F} 11 \mathrm{~A}-\mathrm{C} 8-\mathrm{C} 6$ & $115.3(9)$ \\
\hline $\mathrm{C} 12-\mathrm{Co} 2-\mathrm{Mo} 1$ & $154.70(16)$ & $\mathrm{F} 12 \mathrm{~A}-\mathrm{C} 8-\mathrm{C} 6$ & $111.9(9)$ \\
\hline $\mathrm{C} 11-\mathrm{Co} 2-\mathrm{Mo} 1$ & $99.65(15)$ & $\mathrm{F} 11-\mathrm{C} 8-\mathrm{C} 6$ & $111.6(5)$ \\
\hline $\mathrm{C} 2-\mathrm{Co} 2-\mathrm{Mo} 1$ & $84.29(13)$ & $\mathrm{F} 10-\mathrm{C} 8-\mathrm{C} 6$ & $112.1(5)$ \\
\hline $\mathrm{C} 1-\mathrm{Co} 2-\mathrm{Mo} 1$ & $79.96(12)$ & $\mathrm{F} 12-\mathrm{C} 8-\mathrm{C} 6$ & $112.9(5)$ \\
\hline $\mathrm{S} 3-\mathrm{Co} 2-\mathrm{Mo} 1$ & $56.37(3)$ & $\mathrm{F} 10 \mathrm{~A}-\mathrm{C} 8-\mathrm{C} 6$ & $111.8(9)$ \\
\hline $\mathrm{S} 4-\mathrm{Co} 2-\mathrm{Mo} 1$ & $52.32(3)$ & $\mathrm{O} 1-\mathrm{C} 9-\mathrm{Co} 1$ & $178.5(8)$ \\
\hline $\mathrm{C} 13-\mathrm{Co} 3-\mathrm{C} 14$ & $94.7(2)$ & $\mathrm{O} 2-\mathrm{C} 10-\mathrm{Co} 1$ & $177.0(6)$ \\
\hline $\mathrm{C} 13-\mathrm{Co} 3-\mathrm{S} 4$ & 91.03 (14) & $\mathrm{O} 3-\mathrm{C} 11-\mathrm{Co} 2$ & $177.4(4)$ \\
\hline $\mathrm{C} 14-\mathrm{Co} 3-\mathrm{S} 4$ & $174.25(15)$ & $\mathrm{O} 4-\mathrm{C} 12-\mathrm{Co} 2$ & $176.0(5)$ \\
\hline $\mathrm{C} 13-\mathrm{Co} 3-\mathrm{S} 5$ & $118.60(16)$ & $\mathrm{O} 5-\mathrm{C} 13-\mathrm{Co} 3$ & $176.9(4)$ \\
\hline $\mathrm{C} 14-\mathrm{Co} 3-\mathrm{S} 5$ & $92.28(15)$ & $\mathrm{O} 6-\mathrm{C} 14-\mathrm{Co} 3$ & $177.6(5)$ \\
\hline $\mathrm{S} 4-\mathrm{Co} 3-\mathrm{S} 5$ & $84.38(4)$ & $\mathrm{C} 16-\mathrm{C} 15-\mathrm{C} 20$ & $120.3(4)$ \\
\hline
\end{tabular}




\begin{tabular}{|c|c|c|c|}
\hline $\mathrm{C} 13-\mathrm{Co} 3-\mathrm{S} 6$ & $124.00(15)$ & $\mathrm{C} 16-\mathrm{C} 15-\mathrm{S} 6$ & $118.8(3)$ \\
\hline $\mathrm{C} 14-\mathrm{Co} 3-\mathrm{S} 6$ & $97.07(16)$ & $\mathrm{C} 20-\mathrm{C} 15-\mathrm{S} 6$ & $120.9(3)$ \\
\hline $\mathrm{S} 4-\mathrm{Co} 3-\mathrm{S} 6$ & $80.22(4)$ & $\mathrm{C} 17-\mathrm{C} 16-\mathrm{C} 15$ & $119.5(4)$ \\
\hline $\mathrm{S} 5-\mathrm{Co} 3-\mathrm{S} 6$ & $115.38(4)$ & $\mathrm{C} 17-\mathrm{C} 16-\mathrm{H} 16 \mathrm{~A}$ & 120.2 \\
\hline $\mathrm{C} 13-\mathrm{Co} 3-\mathrm{Mo1}$ & $150.72(14)$ & $\mathrm{C} 15-\mathrm{C} 16-\mathrm{H} 16 \mathrm{~A}$ & 120.2 \\
\hline $\mathrm{C} 14-\mathrm{Co} 3-\mathrm{Mo} 1$ & $114.26(15)$ & $\mathrm{C} 16-\mathrm{C} 17-\mathrm{C} 18$ & $120.8(4)$ \\
\hline $\mathrm{S} 4-\mathrm{Co} 3-\mathrm{Mo} 1$ & $60.00(3)$ & $\mathrm{C} 16-\mathrm{C} 17-\mathrm{H} 17 \mathrm{~A}$ & 119.6 \\
\hline $\mathrm{S} 5-\mathrm{Co} 3-\mathrm{Mo} 1$ & $58.74(3)$ & $\mathrm{C} 18-\mathrm{C} 17-\mathrm{H} 17 \mathrm{~A}$ & 119.6 \\
\hline S6-Co3-Mo1 & $59.27(3)$ & $\mathrm{C} 19-\mathrm{C} 18-\mathrm{C} 17$ & $119.7(4)$ \\
\hline $\mathrm{C} 20-\mathrm{S} 1-\mathrm{Co} 1$ & $98.78(14)$ & $\mathrm{C} 19-\mathrm{C} 18-\mathrm{H} 18 \mathrm{~A}$ & 120.1 \\
\hline $\mathrm{C} 20-\mathrm{S} 1-\mathrm{Mo} 1$ & $106.69(15)$ & $\mathrm{C} 17-\mathrm{C} 18-\mathrm{H} 18 \mathrm{~A}$ & 120.1 \\
\hline $\mathrm{Co} 1-\mathrm{S} 1-\mathrm{Mo} 1$ & $71.67(4)$ & $\mathrm{C} 18-\mathrm{C} 19-\mathrm{C} 20$ & $120.1(4)$ \\
\hline $\mathrm{C} 5-\mathrm{S} 2-\mathrm{Co} 1$ & $97.05(15)$ & $\mathrm{C} 18-\mathrm{C} 19-\mathrm{H} 19 \mathrm{~A}$ & 120.0 \\
\hline $\mathrm{C} 5-\mathrm{S} 2-\mathrm{Mo} 1$ & $106.85(14)$ & $\mathrm{C} 20-\mathrm{C} 19-\mathrm{H} 19 \mathrm{~A}$ & 120.0 \\
\hline $\mathrm{Co} 1-\mathrm{S} 2-\mathrm{Mo} 1$ & $70.94(3)$ & $\mathrm{C} 19-\mathrm{C} 20-\mathrm{C} 15$ & $119.6(4)$ \\
\hline $\mathrm{C} 6-\mathrm{S} 3-\mathrm{Co} 2$ & $101.70(15)$ & $\mathrm{C} 19-\mathrm{C} 20-\mathrm{S} 1$ & $118.7(3)$ \\
\hline $\mathrm{C} 6-\mathrm{S} 3-\mathrm{Mo1}$ & $106.61(14)$ & $\mathrm{C} 15-\mathrm{C} 20-\mathrm{S} 1$ & $121.8(3)$ \\
\hline $\mathrm{Co} 2-\mathrm{S} 3-\mathrm{Mo} 1$ & $73.29(3)$ & & \\
\hline $\mathrm{Co} 3-\mathrm{S} 5-\mathrm{C} 1-\mathrm{C} 2$ & $20.2(3)$ & $\mathrm{Co} 2-\mathrm{S} 3-\mathrm{C} 6-\mathrm{C} 5$ & $79.7(4)$ \\
\hline $\mathrm{Mo} 1-\mathrm{S} 5-\mathrm{C} 1-\mathrm{C} 2$ & $-49.3(3)$ & $\mathrm{Mo1}-\mathrm{S} 3-\mathrm{C} 6-\mathrm{C} 5$ & $3.9(4)$ \\
\hline $\mathrm{Co} 3-\mathrm{S} 5-\mathrm{C} 1-\mathrm{C} 3$ & $-129.4(3)$ & $\mathrm{Co} 2-\mathrm{S} 3-\mathrm{C} 6-\mathrm{C} 8$ & $-101.5(3)$ \\
\hline $\mathrm{Mo} 1-\mathrm{S} 5-\mathrm{C} 1-\mathrm{C} 3$ & $161.1(3)$ & $\mathrm{Mo1}-\mathrm{S} 3-\mathrm{C} 6-\mathrm{C} 8$ & $-177.3(3)$ \\
\hline $\mathrm{Co} 3-\mathrm{S} 5-\mathrm{C} 1-\mathrm{Co} 2$ & $92.78(16)$ & $\mathrm{C} 6-\mathrm{C} 5-\mathrm{C} 7-\mathrm{F} 8 \mathrm{~A}$ & $153.6(10)$ \\
\hline $\mathrm{Mo} 1-\mathrm{S} 5-\mathrm{C} 1-\mathrm{Co} 2$ & $23.27(17)$ & $\mathrm{S} 2-\mathrm{C} 5-\mathrm{C} 7-\mathrm{F} 8 \mathrm{~A}$ & $-26.0(11)$ \\
\hline $\mathrm{C} 3-\mathrm{C} 1-\mathrm{C} 2-\mathrm{C} 4$ & $4.9(7)$ & $\mathrm{C} 6-\mathrm{C} 5-\mathrm{C} 7-\mathrm{F} 7$ & $-37.8(8)$ \\
\hline $\mathrm{S} 5-\mathrm{C} 1-\mathrm{C} 2-\mathrm{C} 4$ & $-140.2(4)$ & $\mathrm{S} 2-\mathrm{C} 5-\mathrm{C} 7-\mathrm{F} 7$ & $142.6(5)$ \\
\hline $\mathrm{Co} 2-\mathrm{C} 1-\mathrm{C} 2-\mathrm{C} 4$ & $121.8(4)$ & $\mathrm{C} 6-\mathrm{C} 5-\mathrm{C} 7-\mathrm{F} 9$ & $84.4(7)$ \\
\hline $\mathrm{C} 3-\mathrm{C} 1-\mathrm{C} 2-\mathrm{S} 4$ & $156.5(3)$ & $\mathrm{S} 2-\mathrm{C} 5-\mathrm{C} 7-\mathrm{F} 9$ & $-95.2(5)$ \\
\hline $\mathrm{S} 5-\mathrm{C} 1-\mathrm{C} 2-\mathrm{S} 4$ & $11.4(4)$ & $\mathrm{C} 6-\mathrm{C} 5-\mathrm{C} 7-\mathrm{F} 7 \mathrm{~A}$ & $-87.7(11)$ \\
\hline $\mathrm{Co} 2-\mathrm{C} 1-\mathrm{C} 2-\mathrm{S} 4$ & $-86.6(3)$ & $\mathrm{S} 2-\mathrm{C} 5-\mathrm{C} 7-\mathrm{F} 7 \mathrm{~A}$ & $92.7(10)$ \\
\hline $\mathrm{C} 3-\mathrm{C} 1-\mathrm{C} 2-\mathrm{Co} 2$ & $-116.9(4)$ & $\mathrm{C} 6-\mathrm{C} 5-\mathrm{C} 7-\mathrm{F} 8$ & $-157.0(6)$ \\
\hline $\mathrm{S} 5-\mathrm{C} 1-\mathrm{C} 2-\mathrm{Co} 2$ & $98.0(3)$ & $\mathrm{S} 2-\mathrm{C} 5-\mathrm{C} 7-\mathrm{F} 8$ & $23.4(6)$ \\
\hline $\mathrm{Co} 3-\mathrm{S} 4-\mathrm{C} 2-\mathrm{C} 1$ & $-37.5(3)$ & $\mathrm{C} 6-\mathrm{C} 5-\mathrm{C} 7-\mathrm{F} 9 \mathrm{~A}$ & $31.1(11)$ \\
\hline $\mathrm{Mo} 1-\mathrm{S} 4-\mathrm{C} 2-\mathrm{C} 1$ & $32.0(3)$ & $\mathrm{S} 2-\mathrm{C} 5-\mathrm{C} 7-\mathrm{F} 9 \mathrm{~A}$ & $-148.5(9)$ \\
\hline $\mathrm{Co} 2-\mathrm{S} 4-\mathrm{C} 2-\mathrm{C} 1$ & $70.6(3)$ & $\mathrm{C} 5-\mathrm{C} 6-\mathrm{C} 8-\mathrm{F} 11 \mathrm{~A}$ & $45.7(14)$ \\
\hline $\mathrm{Co} 3-\mathrm{S} 4-\mathrm{C} 2-\mathrm{C} 4$ & $116.8(3)$ & $\mathrm{S} 3-\mathrm{C} 6-\mathrm{C} 8-\mathrm{F} 11 \mathrm{~A}$ & $-133.1(14)$ \\
\hline $\mathrm{Mo} 1-\mathrm{S} 4-\mathrm{C} 2-\mathrm{C} 4$ & $-173.7(3)$ & $\mathrm{C} 5-\mathrm{C} 6-\mathrm{C} 8-\mathrm{F} 12 \mathrm{~A}$ & $-74.8(13)$ \\
\hline $\mathrm{Co} 2-\mathrm{S} 4-\mathrm{C} 2-\mathrm{C} 4$ & $-135.1(4)$ & $\mathrm{S} 3-\mathrm{C} 6-\mathrm{C} 8-\mathrm{F} 12 \mathrm{~A}$ & $106.4(13)$ \\
\hline $\mathrm{Co} 3-\mathrm{S} 4-\mathrm{C} 2-\mathrm{Co} 2$ & $-108.11(14)$ & $\mathrm{C} 5-\mathrm{C} 6-\mathrm{C} 8-\mathrm{F} 11$ & $78.1(8)$ \\
\hline $\mathrm{Mo} 1-\mathrm{S} 4-\mathrm{C} 2-\mathrm{Co} 2$ & $-38.65(16)$ & $\mathrm{S} 3-\mathrm{C} 6-\mathrm{C} 8-\mathrm{F} 11$ & $-100.7(7)$ \\
\hline $\mathrm{C} 2-\mathrm{C} 1-\mathrm{C} 3-\mathrm{F} 3$ & $36.6(6)$ & $\mathrm{C} 5-\mathrm{C} 6-\mathrm{C} 8-\mathrm{F} 10$ & $-161.6(7)$ \\
\hline $\mathrm{S} 5-\mathrm{C} 1-\mathrm{C} 3-\mathrm{F} 3$ & $-176.6(3)$ & $\mathrm{S} 3-\mathrm{C} 6-\mathrm{C} 8-\mathrm{F} 10$ & $19.6(8)$ \\
\hline $\mathrm{Co} 2-\mathrm{C} 1-\mathrm{C} 3-\mathrm{F} 3$ & $-47.6(5)$ & $\mathrm{C} 5-\mathrm{C} 6-\mathrm{C} 8-\mathrm{F} 12$ & $-42.4(9)$ \\
\hline $\mathrm{C} 2-\mathrm{C} 1-\mathrm{C} 3-\mathrm{F} 1$ & $157.2(4)$ & $\mathrm{S} 3-\mathrm{C} 6-\mathrm{C} 8-\mathrm{F} 12$ & $138.8(7)$ \\
\hline $\mathrm{S} 5-\mathrm{C} 1-\mathrm{C} 3-\mathrm{F} 1$ & $-55.9(4)$ & $\mathrm{C} 5-\mathrm{C} 6-\mathrm{C} 8-\mathrm{F} 10 \mathrm{~A}$ & $165.8(14)$ \\
\hline $\mathrm{Co} 2-\mathrm{C} 1-\mathrm{C} 3-\mathrm{F} 1$ & $73.1(5)$ & $\mathrm{S} 3-\mathrm{C} 6-\mathrm{C} 8-\mathrm{F} 10 \mathrm{~A}$ & $-13.0(14)$ \\
\hline $\mathrm{C} 2-\mathrm{C} 1-\mathrm{C} 3-\mathrm{F} 2$ & $-84.5(5)$ & $\mathrm{Co} 3-\mathrm{S} 6-\mathrm{C} 15-\mathrm{C} 16$ & $-108.6(3)$ \\
\hline
\end{tabular}




\begin{tabular}{|c|c|c|c|}
\hline $\mathrm{S} 5-\mathrm{C} 1-\mathrm{C} 3-\mathrm{F} 2$ & $62.4(4)$ & Mo1-S6-C15-C16 & $-179.2(3)$ \\
\hline $\mathrm{Co} 2-\mathrm{C} 1-\mathrm{C} 3-\mathrm{F} 2$ & $-168.6(3)$ & $\mathrm{Co} 3-\mathrm{S} 6-\mathrm{C} 15-\mathrm{C} 20$ & $71.1(4)$ \\
\hline $\mathrm{C} 1-\mathrm{C} 2-\mathrm{C} 4-\mathrm{F} 4$ & $-69.7(6)$ & Mo1-S6-C15-C20 & $0.5(4)$ \\
\hline $\mathrm{S} 4-\mathrm{C} 2-\mathrm{C} 4-\mathrm{F} 4$ & $138.9(3)$ & $\mathrm{C} 20-\mathrm{C} 15-\mathrm{C} 16-\mathrm{C} 17$ & $-2.5(7)$ \\
\hline $\mathrm{Co} 2-\mathrm{C} 2-\mathrm{C} 4-\mathrm{F} 4$ & $20.4(6)$ & $\mathrm{S} 6-\mathrm{C} 15-\mathrm{C} 16-\mathrm{C} 17$ & $177.3(4)$ \\
\hline $\mathrm{C} 1-\mathrm{C} 2-\mathrm{C} 4-\mathrm{F} 6$ & $170.9(4)$ & $\mathrm{C} 15-\mathrm{C} 16-\mathrm{C} 17-\mathrm{C} 18$ & $2.0(8)$ \\
\hline $\mathrm{S} 4-\mathrm{C} 2-\mathrm{C} 4-\mathrm{F} 6$ & $19.5(5)$ & $\mathrm{C} 16-\mathrm{C} 17-\mathrm{C} 18-\mathrm{C} 19$ & $-0.9(8)$ \\
\hline $\mathrm{Co} 2-\mathrm{C} 2-\mathrm{C} 4-\mathrm{F} 6$ & $-99.0(4)$ & $\mathrm{C} 17-\mathrm{C} 18-\mathrm{C} 19-\mathrm{C} 20$ & $0.3(7)$ \\
\hline $\mathrm{C} 1-\mathrm{C} 2-\mathrm{C} 4-\mathrm{F} 5$ & $52.1(6)$ & $\mathrm{C} 18-\mathrm{C} 19-\mathrm{C} 20-\mathrm{C} 15$ & $-0.8(7)$ \\
\hline $\mathrm{S} 4-\mathrm{C} 2-\mathrm{C} 4-\mathrm{F} 5$ & $-99.3(4)$ & $\mathrm{C} 18-\mathrm{C} 19-\mathrm{C} 20-\mathrm{S} 1$ & $177.5(4)$ \\
\hline $\mathrm{Co} 2-\mathrm{C} 2-\mathrm{C} 4-\mathrm{F} 5$ & $142.3(3)$ & $\mathrm{C} 16-\mathrm{C} 15-\mathrm{C} 20-\mathrm{C} 19$ & $1.9(6)$ \\
\hline $\mathrm{Co} 1-\mathrm{S} 2-\mathrm{C} 5-\mathrm{C} 6$ & $73.6(4)$ & $\mathrm{S} 6-\mathrm{C} 15-\mathrm{C} 20-\mathrm{C} 19$ & $-177.9(3)$ \\
\hline $\mathrm{Mo} 1-\mathrm{S} 2-\mathrm{C} 5-\mathrm{C} 6$ & $1.4(4)$ & $\mathrm{C} 16-\mathrm{C} 15-\mathrm{C} 20-\mathrm{S} 1$ & $-176.3(3)$ \\
\hline $\mathrm{Co} 1-\mathrm{S} 2-\mathrm{C} 5-\mathrm{C} 7$ & $-106.8(3)$ & $\mathrm{S} 6-\mathrm{C} 15-\mathrm{C} 20-\mathrm{S} 1$ & $3.9(5)$ \\
\hline $\mathrm{Mo} 1-\mathrm{S} 2-\mathrm{C} 5-\mathrm{C} 7$ & $-178.9(3)$ & $\mathrm{Co} 1-\mathrm{S} 1-\mathrm{C} 20-\mathrm{C} 19$ & $-111.0(3)$ \\
\hline $\mathrm{C} 7-\mathrm{C} 5-\mathrm{C} 6-\mathrm{C} 8$ & $-2.0(7)$ & Mo1-S1-C20-C19 & $175.6(3)$ \\
\hline $\mathrm{S} 2-\mathrm{C} 5-\mathrm{C} 6-\mathrm{C} 8$ & $177.6(3)$ & $\mathrm{Co} 1-\mathrm{S} 1-\mathrm{C} 20-\mathrm{C} 15$ & $67.2(4)$ \\
\hline $\mathrm{C} 7-\mathrm{C} 5-\mathrm{C} 6-\mathrm{S} 3$ & $176.7(4)$ & $\mathrm{Mo} 1-\mathrm{S} 1-\mathrm{C} 20-\mathrm{C} 15$ & $-6.2(4)$ \\
\hline $\mathrm{S} 2-\mathrm{C} 5-\mathrm{C} 6-\mathrm{S} 3$ & $-3.7(6)$ & & \\
\hline
\end{tabular}

Hydrogen-bond geometry $\left(\AA,{ }^{\circ}\right)$

\begin{tabular}{lllll}
\hline$D-\mathrm{H} \cdots A$ & $D-\mathrm{H}$ & $\mathrm{H} \cdots A$ & $D \cdots A$ & $D-\mathrm{H} \cdots A$ \\
\hline $\mathrm{C} 17-\mathrm{H} 17 A \cdots \mathrm{O}^{\mathrm{i}}$ & 0.95 & 2.57 & $3.353(7)$ & 140 \\
$\mathrm{C} 17-\mathrm{H} 17 A \cdots \mathrm{F} 11^{\mathrm{ii}}$ & 0.95 & 2.62 & $3.461(9)$ & 148 \\
$\mathrm{C} 17-\mathrm{H} 17 A \cdots \mathrm{F} 10 A^{\mathrm{ii}}$ & 0.95 & 2.55 & $3.29(2)$ & 135 \\
\hline
\end{tabular}

Symmetry codes: (i) $-x+1,-y+2,-z$; (ii) $x+1 / 2, y+1 / 2, z$. 